\title{
Molecular Approach to Uterine Leiomyosarcoma: LMP2-Deficient Mice as an Animal Model of Spontaneous Uterine Leiomyosarcoma
}

\author{
Takuma Hayashi, ${ }^{1,2}$ Akiko Horiuchi, ${ }^{3}$ Kenji Sano, ${ }^{4}$ Nobuyoshi Hiraoka, ${ }^{5}$ Yae Kanai, ${ }^{5}$ \\ Tanri Shiozawa, ${ }^{3}$ Susumu Tonegawa, ${ }^{6}$ and Ikuo Konishi ${ }^{7}$ \\ ${ }^{1}$ Department of Immunology and Infectious Disease, Shinshu University Graduate School of Medicine, 3-1-1, Asahi Matsumoto, \\ Nagano 390-8621, Japan \\ ${ }^{2}$ Promoting Business Using Advanced Technology, Japan Science and Technology Agency (JST), Saitama 332-0012, Japan \\ ${ }^{3}$ Department of Obstetrics and Gynecology, Shinshu University School of Medicine, Nagano 390-8621, Japan \\ ${ }^{4}$ Department of Laboratory Medicine, Shinshu University Hospital, Nagano 390-8621, Japan \\ ${ }^{5}$ Pathology Division, National Cancer Center Research Institute, Tokyo 104-0045, Japan \\ ${ }^{6}$ Picower Institute and Department of Biology, Massachusetts Institute of Technology, Cambridge, MA 02139-4307, USA \\ ${ }^{7}$ Department of Gynecology and Obstetrics, Kyoto University Graduate School of Medicine, Kyoto 606-8501, Japan
}

Correspondence should be addressed to Takuma Hayashi, takumah@shinshu-u.ac.jp

Received 16 September 2010; Revised 4 December 2010; Accepted 28 December 2010

Academic Editor: Peter Houghton

Copyright () 2011 Takuma Hayashi et al. This is an open access article distributed under the Creative Commons Attribution License, which permits unrestricted use, distribution, and reproduction in any medium, provided the original work is properly cited.

Uterine leiomyosarcoma (LMS) develops more often in the muscle tissue layer of the uterine body than in the uterine cervix. The development of gynecologic tumors is often correlated with female hormone secretion; however, the development of uterine LMS is not substantially correlated with hormonal conditions, and the risk factors are not yet known. Importantly, a diagnosticbiomarker which distinguishes malignant LMS from benign tumor leiomyoma (LMA) is yet to be established. Accordingly, it is necessary to analyze risk factors associated with uterine LMS, in order to establish a treatment method. LMP2-deficient mice spontaneously develop uterine LMS, with a disease prevalence of $\sim 40 \%$ by 14 months of age. We found LMP2 expression to be absent in human LMS, but present in human LMA. Therefore, defective LMP2 expression may be one of the risk factors for LMS. LMP2 is a potential diagnostic-biomarker for uterine LMS, and may be targeted-molecule for a new therapeutic approach.

\section{Introduction}

The uterus is the female reproductive organ, located at the center of the pelvis between the left and right ovaries. The uterus, the organ in which the embryo grows, is composed of three layers, the uterine endometrium which serves as a bed for the embryo; the myometrium of the wall which protects the embryo; and a serous membrane enveloping the uterus. The myometrium is composed of smooth muscle. In general, the term uterine tumor refers to an epithelial malignant tumor of the uterus, which is roughly classified as a tumor of the uterine cervix or the uterine body. Because of the prevalence of screening, uterine cervix cancer is decreasing in incidence, and usually detected at a very early stage, including stage 0 . In contrast, cancer of the uterine body is increasing in incidence, and rarely detected at the initial stages. While most tumors of the uterine body are adenocarcinomas (derived from the subintimal gland), tumors of the uterine cervix are classified into squamous cancer and adenocarcinoma. The myometrium is composed of smooth muscle. Smooth muscle tumors (SMTs) which develop in the myometrium have been traditionally divided into benign LMA and malignant LMS based on cytological atypia, mitotic activity and other criteria. Uterine LMS, one of the most common neoplasms of the female genital tract, is relatively rare, having an estimated annual incidence of 
0.64 per 100,000 women [1]. Uterine LMS accounts for $2 \%$ to $5 \%$ of tumors of the uterine body and develops more often in the muscle layer of the uterine body than in the uterine cervix [2, 3]. Distinguishing uterine LMA from uterine LMS is very difficult, and a diagnosis generally requires surgery and cytoscopy.

The cause of tumors of the uterine cervix has been found to be the human papilloma virus, in combination with other factors. An infection is established by sexual activity. In contrast, a main factor in the development of tumors in the uterine body is the hormonal environment. Patients with uterine body tumors often are unmarried, have never been pregnant, and are taking a hormonal agent. High estrogen levels are considered to significantly influence the development of such tumors. The mechanisms by which uterine LMA and LMS develop are not yet known, though tumor cells that have developed in the myometrium for some reason gradually become larger due to the influence of the female hormone, estrogen, and generate tumors. However, no correlation between the development of uterine LMS and hormonal conditions, and no obvious risk factors have been found. The prognosis of uterine LMS is not good, and the five-year survival rate is approximately 35\%, although the five-year survival rate depends on disease stage $[2,3]$. It is worth noting that, when adjusted for stage and mitotic count, LMS has a significantly worse prognosis than carcinosarcoma [4]. As uterine LMS is resistant to chemotherapy and radiotherapy, and thus surgical intervention is virtually the only means of treatment [5-7], developing an efficient adjuvant therapy is expected to improve the prognosis of the disease. Although cases accompanied by hypocalcaemia or eosinophilia have been reported, neither clinical abnormality is an initial risk factor for uterine LMS. The identification of a risk factor associated with the development of uterine LMS would significantly contribute to the development of preventive and therapeutic treatments.

\section{Biological Roles of the Immunoproteasome}

When tissue or an organ is transplanted, the graft is often lost due to an acute rejection caused by the host immune system. This is because the cell surface antigens presented by the major histocompatibility complex (MHC) are intrinsic to an individual and so differ between the donor and recipient. The immunological self markers on cell surfaces are the most important immune system for higher vertebrates such as mammals, protecting the self from invaders. Cytoplasmic proteins are mostly degraded by a protease complex, which has many substrates consisting of twenty-eight 20 to 30$\mathrm{kDa}$ subunits, referred to as the immunoproteasome [8]. The proteasomal degradation pathway is essential for many cellular processes, including the cell cycle, the regulation of gene expression, and others. The proteasomal degradation pathway is also essential for the production of peptide antigens which are presented by MHC class I. That is, the immunoproteasome plays a key role in the presentation of immunological self markers on the cell surface by MHC (Figure 1) [8]. Interferon- $\gamma($ IFN-) $\gamma$ ) is a critical inducer of

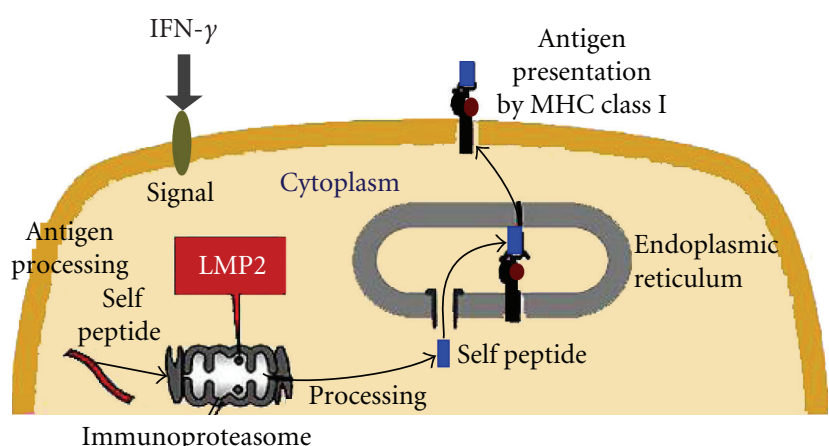

FIGURE 1: Mediation of the proteasomal degradation pathway to antigen presentation by MHC class I. The immunoproteasomal degradation pathway is essential for antigen presentation by MHC class I. Defecive LMP2 expression results in tissue- and substratedependent abnormalities of immunoproteasomal functions. Therefore an impaired proteasome may promote the initial development of disease including tumorigenesis.

the immunoproteasome's expression in immune systems [9]. Recent findings have verified that IFN- $\gamma$ prevents primary tumor development, thereby showing a tumor suppressor role in the immune response $[10,11]$. IFN $-\gamma$ upregulates the expression of large numbers of responsive genes, also, expression of the immunoproteasome's subunits, that is, lowmolecular mass polypeptide (LMP) 2, LMP7, and LMP10, is markedly induced by IFN- $\gamma$ signaling $[9,12]$. The IFN- $\gamma$ inducible proteasomal function plays a key role in MHC class I-mediated tumor rejection [11, 13]. Further, a molecular approach to studying the correlation of IFN- $\gamma$ with tumor cell growth has drawn attention. A deficiency of IFN- $\gamma$ apparently does not hamper the generation of CTL [10, 11]. Recent reports have demonstrated the multifunctional deficiencies of components of the MHC class I antigenpresentation pathway including LMP2 and TAP-1 in tumor cells $[11,13]$. A possible role for the IFN- $\gamma$-responsive gene TAP-1 in tumor recognition was reported [11]. Here we identify LMP2, a single IFN- $\gamma$-responsive gene product, as obligatory for tumor surveillance [12] and demonstrate a tissue-specific role for LMP2 in protection from spontaneous neoplasms of the uterus.

\section{Development of Malignant Uterine Tumor in LMP2-Deficient Mice}

Malignant tumors originate from a single cancerous cell and develop as a result of unlimited cell proliferation. Malignant tumor cells have properties that are biologically different from those of normal cells. Thus, the host immune system should be able to distinguish malignant tumor cells from corresponding normal cells. That is, malignant tumor cells present intrinsic antigens (i.e., tumor-cell-specific antigens that can be the targets of immune responses are referred to as tumor-antigens (TA)) on the cell surface with the aid of MHC. In many cases, however, almost no reaction by the immune system is observed. Also, the incidence of major tumors is not very different between immunodeficient 


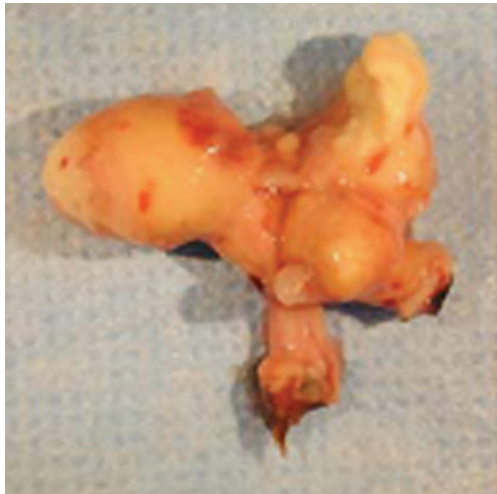

(a)

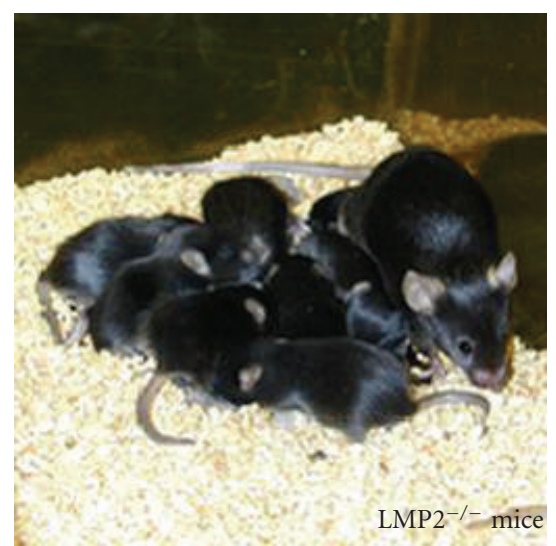

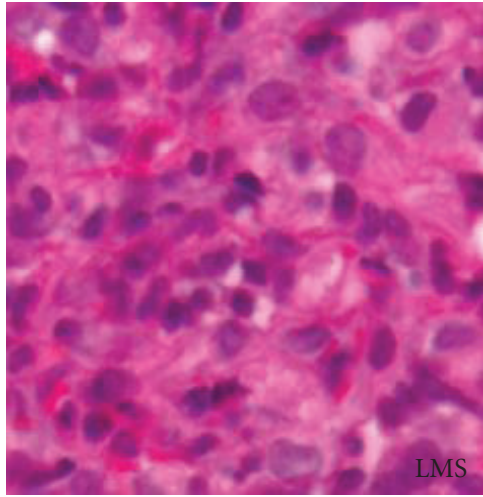

(b)

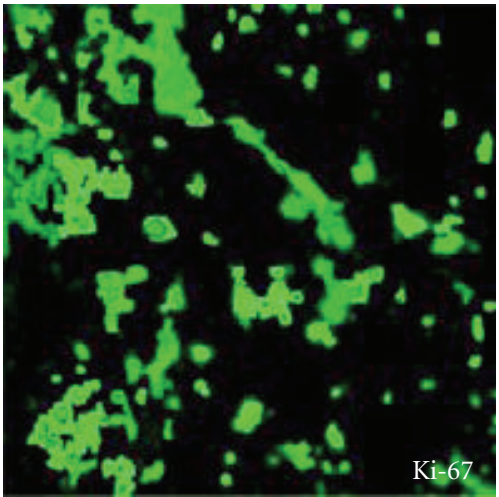

(c)

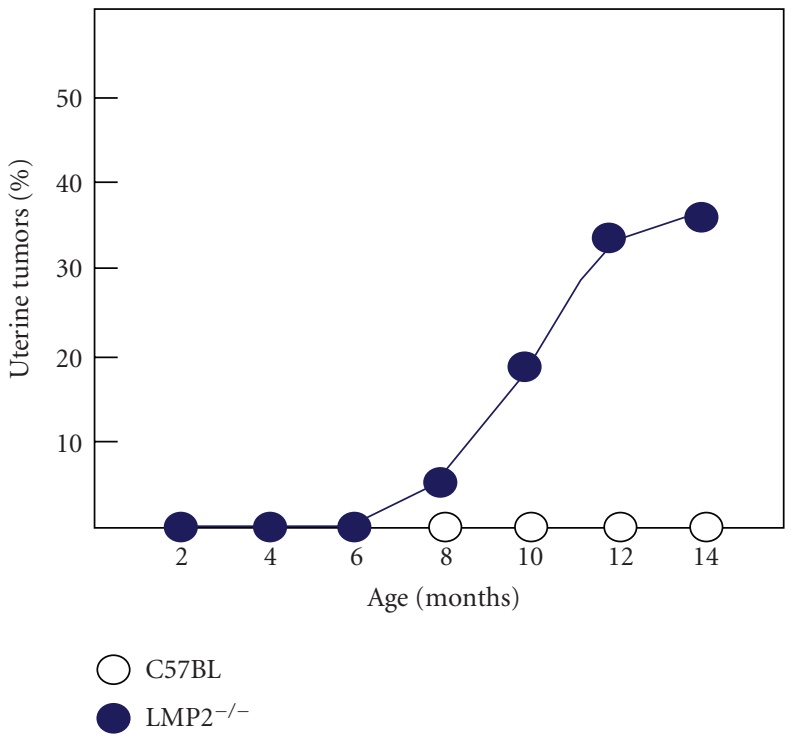

(e)

Figure 2: Histological findings of uterine leiomyosarcoma in LMP2-deficient mice. Histological findings of uterine LMS in LMP2-deficient mice ((a) to (c)). Among the histological findings of uterine LMS in LMP2-deficient mice, a cytoskeleton, which is characteristic of uterine LMS, is observed. ((b) and (c) magnification x400) Panel (e), in LMP2-deficient females, uterine LMS is observed at 6 months of age. The incidence at age 14 months is as high as $40 \%$ (e). The curve indicating the incidence of mouse uterine LMS is very similar to that indicating the incidence of human uterine LMS, which is observed after menopause. In mice with tumors of the uterus, significant weight loss is observed. Thus, a tumor that develops in the uterus is diagnosed as malignant, that is, uterine LMS.

(i.e., lymphocyte-deficient) mice and control mice having normal immune systems. Specifically, tumor cells can avoid the immune monitoring system via several means [14, 15]. Naturally occurring tumor cells seem to have lost the expression of peptide antigens, TA, or cell adhesion factors intrinsic to tumors. Tumor cells may avoid the host immune reaction due to the absence of MHC expression, although no such mechanism has yet been elucidated. However, it is important to demonstrate how tumor cells evade immuneresponses, in order to prevent the development of tumors.

The genes encoding LMP2, LMP7, TAP1, and TAP2, are located in region $\mathrm{H}-2$ which encodes the murine MHC molecule. LMP2-deficient mice show tissue- and substratedependent abnormalities in the biological functions of the immunoproteasome, and impaired functioning of the immunoproteasome in the spleen or hepatic cells [16].
Further, LMP2-deficient mice do not show normal immune responses to virus-infected cells, and such immunopathy is known to result from a failure in the presentation of peptide antigens on the cell surface by MHC [16]. We found that uterine LMS occurred in female LMP2-deficient mice at age 6 months or older, and the incidence at 14 months of age was about 40\% [17] (Figure 2). The curve indicating the incidence in mice is very similar to that indicating the incidence of human uterine LMS, which occurs after menopause. Histological examinations of LMP2lacking uterine tumors revealed characteristic abnormalities of LMS [17]. The tumors lacked lymphoid infiltrates, a sign of immune recognition, and consisted of uniform elongated smooth muscle cells arranged into bundles. The nuclei of the tumor cells varied in size and shape; furthermore, mitosis was frequent, in contrast, the uterine smooth muscle cells 
of C57BL/6 mice were normal in appearance [17]. Whereas relatively few Ki-67-positive cells, the proliferating cells of solid tumors, were observed in the basal cell layer of the normal uterine smooth muscle, most of the basal cells vividly expressed Ki-67 in LMP2-deficient mice [17]. This immunohistochemical (IHC) staining indicates abnormal proliferation of the LMP2-lacking cells in the basal layer [17] (Figure 2). LMP2-deficient mice that have developed uterine LMS undergo considerable weight loss, and then die by 14 months of age [17]. The LMP2-deficient mice also exhibit skeletal muscle metastasis from uterine LMS. Therefore it is like LMP2-deficient mice with uterine LMS have died of mass effect and metastasis. In general, it is not easy to distinguish uterine LMA from LMS. However, in mice, because of such characteristic pathological findings, significant weight loss, and exhibition of skeletal muscle metastasis, a tumor that develops in the uterus of an LMP2-deficient mouse can be considered malignant, that is, a uterine LMS.

If the TP53 gene is damaged, tumor suppression is severely reduced. People who inherit only one functional copy of the TP53 gene will most likely develop tumors in early adulthood, a disease known as Li-Fraumeni syndrome. More than 50 percent of human tumors contain a mutation or deletion of the TP53 gene [18]. To increase tumor incidence and better assess the role of systemic expression of TP53 in responses to initiation of uterine LMS tumorigenesis, LMP2-deficient mice were bred with TP53deficient mice to create $L m p 2^{-/-} T p 53^{-/-}$double knockout mice. Uterine LMS incidence and death rates were similar in $L m p 2^{-/-} T p 53^{-/-}$mice and closely matched control $L m p 2^{-/-} \mathrm{Tp} 53^{+/+}$mice. The correlation of defective TP53 function with uterine LMS tumorigenesis is not clearly understood.

\section{Inactivation of the IRF-1 Tumor Suppressor Gene in LMP2-Deficient Mice}

Uterine LMS was demonstrated to spontaneously develop in 6-month-old LMP2-deficient mice at high frequency. The expression of LMP2 was significantly induced by IFN- $\gamma$ as was the expression of other subunits $[9,12]$. Accordingly, the expression of cell-cycle regulators that are regulated by the IFN- $\gamma$ signal cascade or immunoproteasome activity was examined. Signal transducer and activator of transcription (STAT) 1, having been activated by IFN- $\gamma$, significantly induced expression of tumor suppressors such as interferon regulatory factor 1 (IRF1) [19, 20]. IRF1 as a transcriptional regulator significantly regulates LMP2 expression $[19,20]$. It was examined whether the IFN- $\gamma$ signal cascade induces the expression of each subunit of the immunoproteasome and IFR1 and IRF2 in LMP2-deficient mice and the parental strain, C57BL/6. No significant difference was observed in the expression of STAT1 and the subunits LMP7, LMP10, CP9, and IRF2. Also, IFN- $\gamma$-induced phosphorylation of STAT1 would not be influenced by a lack of LMP2. However, the expression of IRF1 was significantly reduced in splenocytes derived from mice lacking LMP2 in comparison with wild-type mice. IRF1 expression in LMP2-deficient

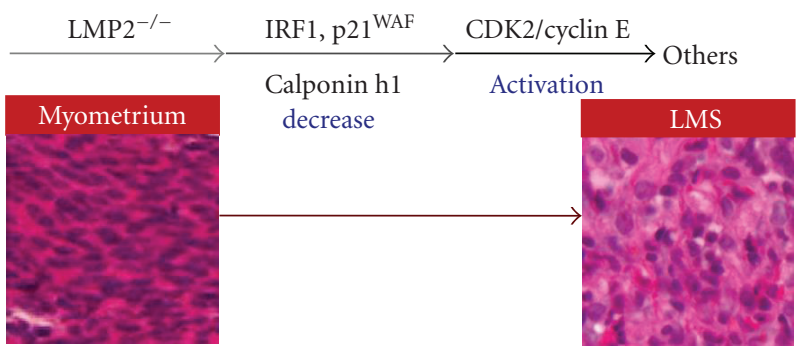

FIgURE 3: Model of the mechanism for development of uterine leiomyosarcoma. In LMP2-deficient cells, levels of the antioncogenic factor IRF-1, p2 $1^{\text {WAF }}$ are significantly reduced. Reduced expression of the calponin h1 transcript, which contributes to cell proliferation and tumorigenesis in uterine smooth muscle cells, is detected in uterine LMS tissues. Cell cycle regulatory factors, CDK2/Cyclin E, are markedly activated. The inactivation of such antioncogenic factors is considered to transform LMP2-deficient cells into malignant tumor cells.

splenocytes was not induced by the IFN- $\gamma$ signal cascade. In addition, wild type-mouse embryonic fibroblasts (MEFs) that had been treated with the proteasome inhibitor MG132 exhibited a loss of IFN- $\gamma$-inducibility, reproducing a phenotype of the LMP2-deficient mouse. Accordingly, the transcription of Irf1 mRNA depends on the immunoproteasome's function and is considered to involve the formation of a STAT1 homodimer. Recent reports suggest that proteasomal function contributes to mRNA transcriptional activation $[21,22]$.

Primary cultured tumor cells (LMP2-UC) were established from the uterine LMS of LMP2-deficient mice, and then IRF1-overexpressing tumor cells (LMP2-UCIRF1) were further established by genetic engineering. The LMP2-UC-IRF1 cells were intracutaneously transplanted into immunodeficient mice (BALB/c nu/nu), and significant inhibiting effects of IRF1 on tumor cell proliferation were observed [20,23]. Thus, a lowered level of IRF1 resulting from a deficiency in LMP2 seemed to be a risk factor for uterine LMS in mice. The effects of IRF1 on tumor cell proliferation are achieved through the expression of $\mathrm{p} 21^{\mathrm{WAF}}$ cell-cycle inhibitors (inhibiting transition from the G1 to S stage) [24]. Whether or not $\mathrm{p} 21^{\mathrm{WAF}}$ expression or activation is affected in LMP2-deficient mice should be examined further. The tumor suppressor, retinoblastoma $(\mathrm{Rb})$ is phosphorylated by a complex of Cyclin E/Cyclin dependent kinase 2 (CDK2) and then inactivated [25]. Also, the activity of CDK2 is negatively regulated via degradation of Cyclin E by the $26 \mathrm{~S}$ proteasome [26, 27]. A significant level of phosphorylated-Rb is observed in MEFs-lacking LMP2, and the activity of CDK2 for phosphorylation is determined to be stronger than that in normal MEFs. However research overall, including experiments with genedeficient mouse models and clinical studies, suggests that defective $\mathrm{Rb}$ expression does not take part in the onset of uterine LMS [28-30]. In the case of uterine LMS in LMP2-deficient mice, defective IRF1 is considered to be involved in cellular transformation and cell proliferation (Figure 3). 


\section{Perspectives}

Uterine LMS mainly develops in the uterine smooth muscle or endometrial stroma, and menstrual anomalies, such as hypermenorrhea and prolonged menstruation, and symptoms such as abnormal hemorrhage, hypogastric pain, lumbar pain, and abdominal strains, are observed [4]. In the case of gynecological cancers, such as breast cancer, a female hormonal imbalance is often a risk factor for developing tumors. As in the case of uterine LMA, however, a correlation between the development of uterine LMS, the female hormone, and hormone receptors has yet to be elucidated [31, 32]. A recent report showed the expression of $L m p 2$ mRNA and protein in luminal and glandular epitheliua, placenta villi, trophoblastic shells, and arterial endothelial cells [33]. These results implicate LMP2 in the invasion of placental villi, degradation of the extracellular matrix, immune tolerance, glandular secretion, and angiogenesis [33]. The present study should help to elucidate the regulatory role of the ubiquitin-proteasome pathway in the implantation of embryos. Unfortunately, it is unclear whether defective LMP2 expression is involved in the onset of uterine LMS. Uterine LMS often seems to develop in individuals exposed to radiation in the pelvis. Risk factors for its development, however, have not been identified because of the absence of a suitable animal model. The LMP2-deficient mouse was the first animal model of spontaneous uterine LMS to be established [17]. Defective LMP2 expression may be one of the causes of uterine LMS [20]. To demonstrate whether LMP2 is a potential biomarker for distinguishing LMS from LMA, we are investigating the reliability and characteristics of LMP2 as a diagnostic indicator with several clinical research facilities. The clinical research is yet to be concluded, and large-scale clinical studies need to be performed. In some cases, uterine LMA may become malignant and develop into uterine LMS. Accordingly, the correlation between the inactivation of LMP2 and the development of uterine LMA needs to be examined. Although LMS usually lacks lymphoid infiltrates recognizable on routine histological staining, further histological examination revealed a few infiltrating $\mathrm{CD}_{56}{ }^{+}$natural killer cells in human uterine LMS tissues. Definitive histological studies must be performed, including the gene-expression profiling of several known pro-oncogenic factors as well as factors such as brain-specific polypeptide PEP-19 and a transmembrane tyrosine kinase receptor, C-kit [34-36]. The reduced expression of calponin h1 transcripts was reported to be associated with uterine LMS, and calponon h1 might function as a tumor suppressor in uterine LMS $[37,38]$. A recent study showed that reexpression of human calponin h1 suppressed cell proliferation and tumorigenesis in uterine LMS cells [38]. Since no spontaneous development of uterine LMS is observed in IRF1-, calponin h1-deficient mice or heterozygous $\mathrm{Rb}$ mice, the lack of LMP2 is largely associated with the expression of other known or unknown cell-cycle regulatory factors. Further research is required to demonstrate the correlative functions of LMP2 and other antioncogenic factors with calponin $\mathrm{h} 1$ in the tumorigenesis of uterine LMS. Clarification of the correlation between these factors and the development of uterine LMS and the identification of specific risk factors may lead to the development of new treatments for the disease. Uterine LMS is refractory to chemotherapy and has a poor prognosis. The molecular biological and cytological information obtained from LMP2deficient mice will contribute remarkably to the development of preventive methods, a potential diagnostic-biomarker, and new therapeutic approaches against uterine LMS.

\section{Acknowledgments}

The authors sincerely thank Professor Luc Van Kaer (Vanderbilt University Medical Center). This study was supported in part by grants from the Ministry of Education, Culture, Science and Technology, and The Foundation of Osaka Cancer Research, The Ichiro Kanehara Foundation for the Promotion of Medical Science and Medical Care, The foundation for the Promotion of Cancer Research, The Kanzawa Medical Research Foundation, The Shinshu Medical Foundation, and The Takeda Foundation for Medical Science.

\section{References}

[1] C. Zaloudek and M. R. Hendrickson, "Mesenchymal tumors of the uterus," in Blaustein's Pathology of the Female Genital Tract, R. J. Kurman, Ed., pp. 561-578, Springer, New York, NY, USA, 5th edition, 2002.

[2] J. F. Lin and B. M. Slomovitz, "Uterine sarcoma 2008," Current Oncology Reports, vol. 10, no. 6, pp. 512-518, 2008.

[3] F. Amant, AN. Coosemans, M. Debiec-Rychter, D. Timmerman, and I. Vergote, "Clinical management of uterine sarcomas," The Lancet Oncology, vol. 10, no. 12, pp. 11881198, 2009.

[4] M. Miettinen and J. F. Fetsch, "Evaluation of biological potential of smooth muscle tumours," Histopathology, vol. 48, no. 1, pp. 97-105, 2006.

[5] S. E. Brooks, M. Zhan, T. Cote, and C. R. Baquet, "Surveillance, epidemiology, and end results analysis of 2677 cases of uterine sarcoma 1989-1999," Gynecologic Oncology, vol. 93, no. 1, pp. 204-208, 2004.

[6] K. E. Dusenbery, R. A. Potish, and P. Judson, "Limitations of adjuvant radiotherapy for uterine sarcomas spread beyond the uterus," Gynecologic Oncology, vol. 94, no. 1, pp. 191-196, 2004.

[7] T. I. Wu, T. C. Chang, S. Hsueh et al., "Prognostic factors and impact of adjuvant chemotherapy for uterine leiomyosarcoma," Gynecologic Oncology, vol. 100, no. 1, pp. 166-172, 2006.

[8] T. Maniatis, "A ubiquitin ligase complex essential for the NF$\kappa \mathrm{B}$, Wnt/Wingless, and Hedgehog signaling pathways," Genes and Development, vol. 13, no. 5, pp. 505-510, 1999.

[9] M. Groettrup, S. Khan, K. Schwarz, and G. Schmidtke, "Interferon- $\gamma$ inducible exchanges of $20 \mathrm{~S}$ proteasome active site subunits: why?" Biochimie, vol. 83, no. 3-4, pp. 367-372, 2001.

[10] C. Nakajima, Y. Uekusa, M. Iwasaki et al., "A role of interferon$\gamma($ IFN- $\gamma)$ in tumor immunity: T cells with the capacity to reject tumor cells are generated but fail to migrate to tumor sites in IFN- $\gamma$-deficient mice," Cancer Research, vol. 61, no. 8, pp. 3399-3405, 2001. 
[11] V. Shankaran, H. Ikeda, A. T. Bruce et al., "IFN $\gamma$, and lymphocytes prevent primary tumour development and shape tumour immunogenicity," Nature, vol. 410, no. 6832, pp. 1107-1111, 2001.

[12] M. Gaczynska, K. L. Rock, and A. L. Goldberg, " $\gamma$-interferon and expression of MHC genes regulate peptide hydrolysis by proteasomes," Nature, vol. 365, no. 6443, pp. 264-267, 1993.

[13] K. Delp, F. Momburg, C. Hilmes, C. Huber, and B. Seliger, "Functional deficiencies of components of the MHC class I antigen pathway in human tumors of epithelial origin," Bone Marrow Transplantation, vol. 25, supplement 2, pp. S88-S95, 2000.

[14] G. P. Dunn, A. T. Bruce, H. Ikeda, L. J. Old, and R. D. Schreiber, "Cancer immunoediting: from immunosurveillance to tumor escape," Nature Immunology, vol. 3, no. 11, pp. 991-998, 2002.

[15] G. P. Dunn, L. J. Old, and R. D. Schreiber, "The immunobiology of cancer immunosurveillance and immunoediting," Immunity, vol. 21, no. 2, pp. 137-148, 2004.

[16] L. Van Kaer, P. G. Ashton-Rickardt, M. Eichelberger et al., "Altered peptidase and viral-specific T cell response in LMP2 mutant mice," Immunity, vol. 1, no. 7, pp. 533-541, 1994.

[17] T. Hayashi and D. L. Faustman, "Development of spontaneous uterine tumors in low molecular mass polypeptide-2 knockout mice," Cancer Research, vol. 62, no. 1, pp. 24-27, 2002.

[18] M. Hollstein, D. Sidransky, B. Vogelstein, and C. C. Harris, "p53 mutations in human cancers," Science, vol. 253, no. 5015, pp. 49-53, 1991.

[19] M. Brucet, L. Marqués, C. Sebastián, J. Lloberas, and A. Celada, "Regulation of murine Tap1 and Lmp2 genes in macrophages by interferon gamma is mediated by STAT 1 and IRF-1," Genes and Immunity, vol. 5, no. 1, pp. 26-35, 2004.

[20] T. Hayashi, Y. Kobayashi, S. Kohsaka, and K. Sano, "The mutation in the ATP-binding region of JAK1, identified in human uterine leiomyosarcomas, results in defective interferon- $\gamma$ inducibility of TAP1 and LMP2," Oncogene, vol. 25, no. 29, pp. 4016-4026, 2006.

[21] S. Yanagi, N. Shimbara, and T. A. Tamura, "Tissue and cell distribution of a mammalian proteasomal ATPase, MSS1, and its complex formation with the basal transcription factors," Biochemical and Biophysical Research Communications, vol. 279, no. 2, pp. 568-573, 2000.

[22] I. Lassot, D. Latreille, E. Rousset et al., "The proteasome regulates HIV-1 transcription by both proteolytic and nonproteolytic mechanisms," Molecular Cell, vol. 25, no. 3, pp. 369383, 2007.

[23] H. Harada, M. Kitagawa, N. Tanaka et al., "Anti-oncogenic and oncogenic potentials of interferon regulatory factors-1 and -2," Science, vol. 259, no. 5097, pp. 971-974, 1993.

[24] N. Tanaka, M. Ishihara, M. S. Lamphier et al., "Cooperation of the tumour suppressors IRF-1 and p53 in response to DNA damage," Nature, vol. 382, no. 6594, pp. 816-818, 1996.

[25] C. J. Sherr, "The pezcoller lecture: cancer cell cycles revisited," Cancer Research, vol. 60, no. 14, pp. 3689-3695, 2000.

[26] D. M. Koepp, L. K. Schaefer, X. Ye et al., "Phosphorylationdependent ubiquitination of cyctin E by the SCF ubiquitin ligase," Science, vol. 294, no. 5540, pp. 173-177, 2001.

[27] Y. Matsumoto and J. L. Maller, "A centrosomal localization signal in cyclin $\mathrm{E}$ required for Cdk2-independent $\mathrm{S}$ phase entry," Science, vol. 306, no. 5697, pp. 885-888, 2004.

[28] S. X. Liang, Y. Lakshmanan, B. A. Woda, and Z. Jiang, "A highgrade primary leiomyosarcoma of the bladder in a survivor of retinoblastoma," Archives of Pathology and Laboratory Medicine, vol. 125, no. 9, pp. 1231-1234, 2001.
[29] L. Venkatraman, J. R. Goepel, K. Steele, S. P. Dobbs, R. W. Lyness, and W. G. McCluggage, "Soft tissue, pelvic, and urinary bladder leiomyosarcoma as second neoplasm following hereditary retinoblastoma," Journal of Clinical Pathology, vol. 56, no. 3, pp. 233-236, 2003.

[30] E. Calo, J. A. Quintero-Estades, P. S. Danielian, S. Nedelcu, S. D. Berman, and J. A. Lees, "Rb regulates fate choice and lineage commitment in vivo," Nature, vol. 466, no. 7310, pp. 1110$1114,2010$.

[31] Y. L. Zhai, Y. Kobayashi, A. Mori et al., "Expression of steroid receptors, Ki-67, and p53 in uterine leiomyosarcomas," International Journal of Gynecological Pathology, vol. 18, no. 1, pp. 20-28, 1999.

[32] S. E. Akhan, E. Yavuz, A. Tecer et al., "The expression of Ki-67, p53, estrogen and progesterone receptors affecting survival in uterine leiomyosarcomas. A clinicopathologic study," Gynecologic Oncology, vol. 99, no. 1, pp. 36-42, 2005.

[33] H. X. Wang, H. M. Wang, Q. L. Li, H. Y. Lin, D. Qian, and C. Zhu, "Expression of proteasome subunits low molecular mass polypeptide (LMP) 2 and LMP7 in the endometrium and placenta of rhesus monkey (Macaca mulatta) during early pregnancy," Biology of Reproduction, vol. 71, no. 4, pp. 13171324, 2004.

[34] T. Kanamori, K. Takakura, M. Mandai et al., "PEP-19 overexpression in human uterine leiomyoma," Molecular Human Reproduction, vol. 9, no. 11, pp. 709-717, 2003.

[35] L. Wang, J. C. Felix, J. L. Lee et al., "The proto-oncogene ckit is expressed in leiomyosarcomas of the uterus," Gynecologic Oncology, vol. 90, no. 2, pp. 402-406, 2003.

[36] S. K. Ylisaukko-Oja, M. Kiuru, H. J. Lehtonen et al., "Analysis of fumarate hydratase mutations in a population-based series of early onset uterine leiomyosarcoma patients," International Journal of Cancer, vol. 119, no. 2, pp. 283-287, 2006.

[37] A. Horiuchi, T. Nikaido, K. Ito et al., "Reduced expression of calponin h1 in leiomyosarcoma of the uterus," Laboratory Investigation, vol. 78, no. 7, pp. 839-846, 1998.

[38] A. Horiuchi, T. Nikaido, S. Taniguchi, and S. Fujii, "Possible role of calponin h1 as a tumor suppressor in human uterine leiomyosarcoma," Journal of the National Cancer Institute, vol. 91, no. 9, pp. 790-796, 1999. 


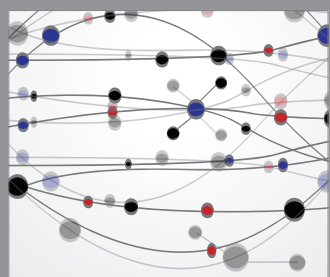

The Scientific World Journal
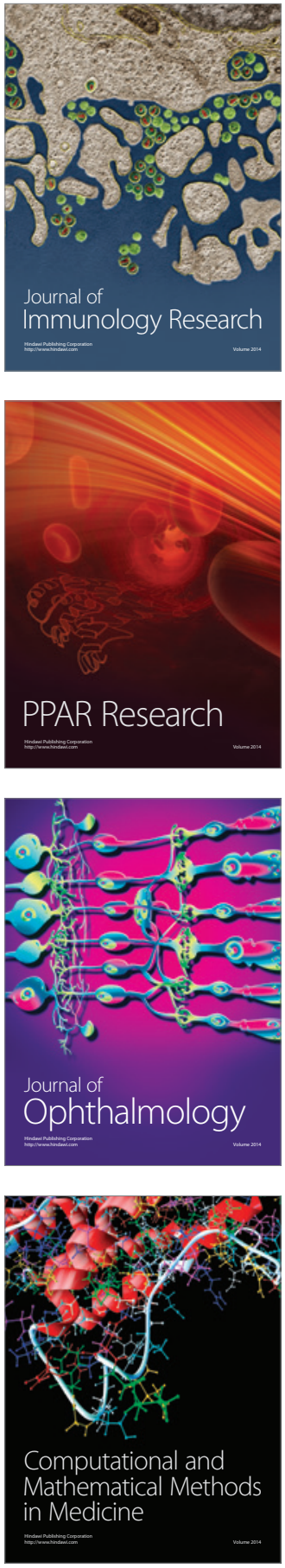

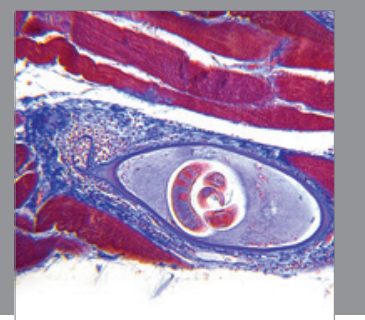

Gastroenterology

Research and Practice
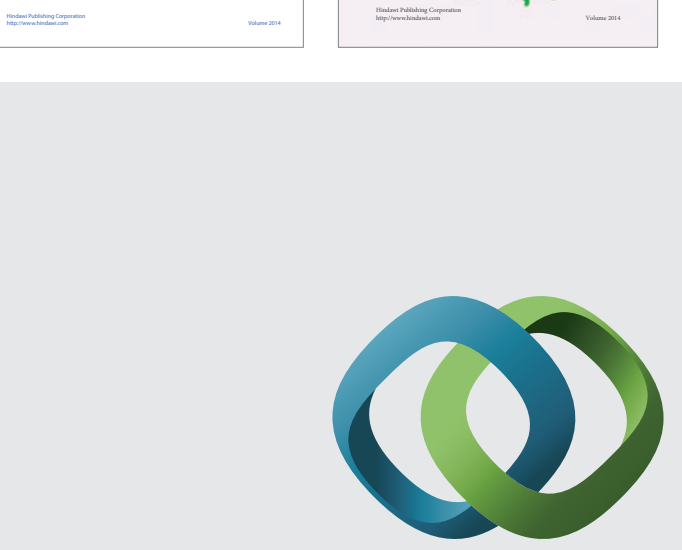

\section{Hindawi}

Submit your manuscripts at

http://www.hindawi.com
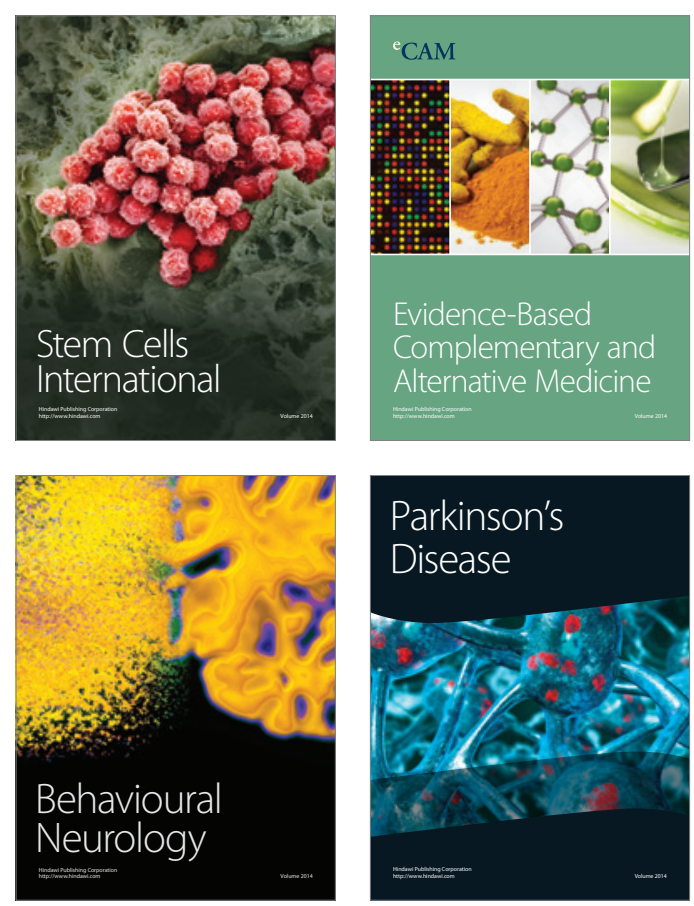

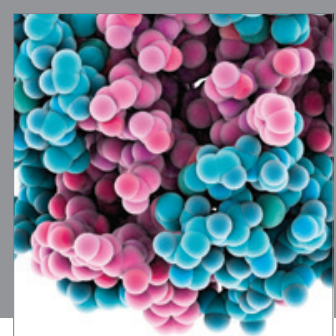

Journal of
Diabetes Research

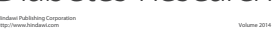

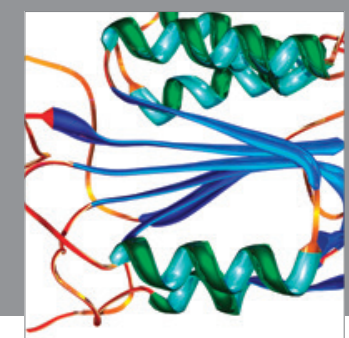

Disease Markers
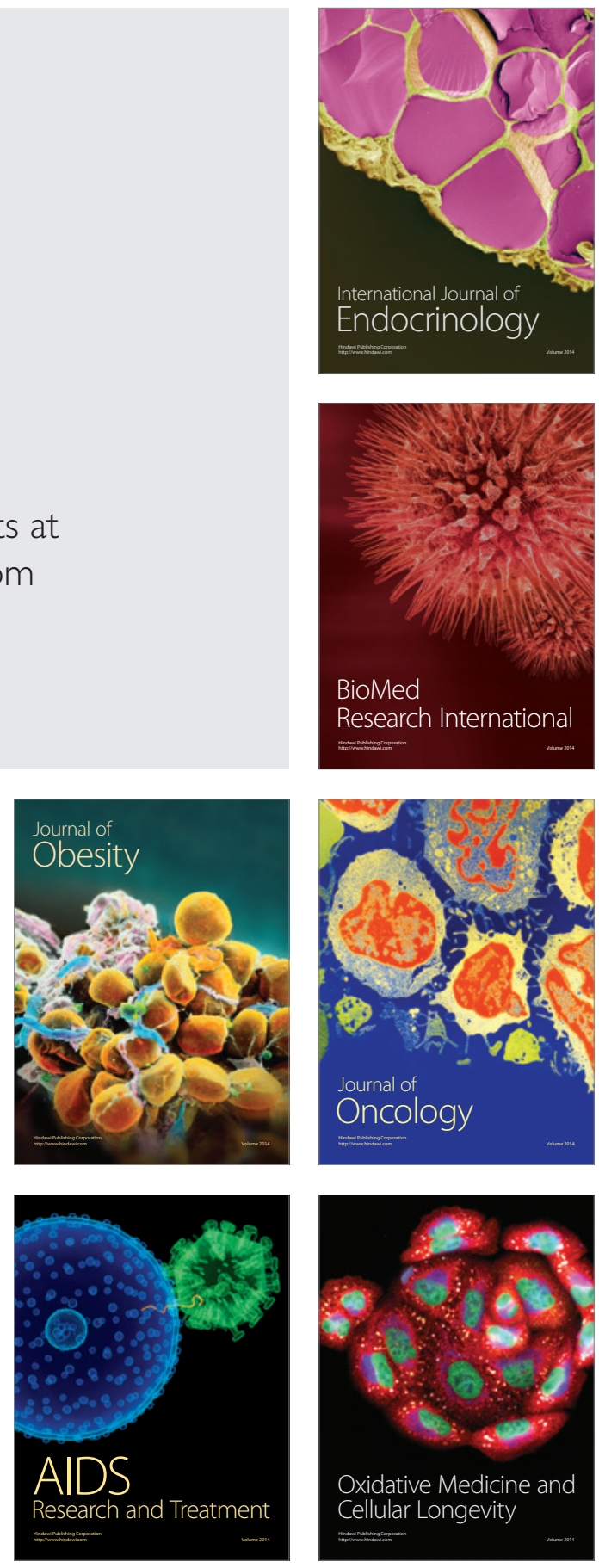